\title{
Coder perspectives on physician-related barriers to producing high-quality administrative data: a qualitative study
}

\author{
Karen L. Tang MD, Kelsey Lucyk MSc, Hude Quan MD PhD \\ See related commentary by Nicholls and colleagues at www.cmaj.ca/content/189/33/E1054
}

\section{Abstract}

Background: Professional coding specialists ("coders") are experts at translating patient chart information into alphanumerical codes, which are then widely used in research and health policy decision-making. Coders rely solely on documentation by health care providers to complete this task. We aimed to explore physician-related barriers to coding that results in high-quality administrative data.

Methods: In a qualitative study conducted from December 2015 to March 2016, we recruited 28 coders who worked in health care facilities in Alberta using purposive and snowball sampling. Semistructured interviews were conducted, audio-recorded and transcribed. The interviews delved into coder training, work environment, documentation and coding standards. Thematic content analysis of transcripts was performed by 2 study investigators through line-by-line coding and constant comparison, after which the codes were collated into themes.

Results: Five themes emerged regarding physician-related barriers in coding of high-quality administrative data: 1) coders are limited in their ability to add to, modify or interpret physician documentation, which supersedes all other chart documentation, 2) physician documentation is incomplete and nonspecific, 3) chart information tends to be replete with errors and discrepancies, 4) physicians and coders use different terminology to describe clinical diagnoses and 5) there is a communication divide between coders and physicians, such that questions and issues regarding physician documentation cannot be reconciled.

Interpretation: Physicians play a major role in influencing the quality of administrative data. There is a need for physicians to advocate for culture change in physicians' attitudes toward coders and chart documentation, in recognition of the importance of accurate chart information.

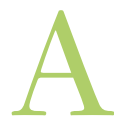

dministrative data are information collected to carry out decisions rather than for primary research purposes. An example is the Discharge Abstract Database, which captures information on hospital admissions. Throughout a patient's hospital stay, health care providers document the events of the entire stay through progress notes and the narrative discharge summary in the clinical chart. ${ }^{1}$ On discharge, this chart is transferred to the health records department, where professional coding specialists ("coders") translate the hospital stay into alphanumerical data that capture patient diagnoses, procedures, services used and complications. Medical diagnoses are coded with the use of a standard classification system such as the International Classification of Disease, 10th Revision (ICD-10). These data are then widely used in research ${ }^{2,3}$ and disease surveillance ${ }^{4}$ and to evaluate indicators of the quality of health care delivery. ${ }^{3}$ High-quality administrative data, with accurate, specific, comprehensive and precise diagnostic codes, are therefore important for both research and decision-making. ${ }^{5}$ However, known quality issues exist, especially with nonspecificity and imprecision., ${ }^{3,6-10}$

Most studies on barriers to coding that result in highquality administrative data have focused on coder-related barriers concerning their training, knowledge and evaluation standards. ${ }^{11-17}$ One barrier that is consistently described by coders is the lack of precise documentation in clinical charts, ${ }^{1,13,18}$ which accounts for half of the disagreements in diagnostic codes among coders. ${ }^{10}$ Although physicians provide

\section{Competing interests: None declared.}

This article has been peer reviewed.

Correspondence to: Karen Tang, klktang@ucalgary.ca

CMAJ Open 2017. DOI:10.9778/cmajo.20170036 
the main sources of chart documentation, the role that they play in determining the quality of coded data remains unknown. The objective of this qualitative study was to identify the barriers to coding resulting in high-quality administrative data from the perspective of coders and the role that physicians play in contributing to these barriers.

\section{Methods}

\section{Data collection}

We used a multimodal recruitment strategy that included purposive and snowball sampling. ${ }^{19,20}$ The only inclusion criterion for study participation was that the person be a coding specialist working in a health care facility in Alberta. Of note, all coders working in Canada must be certified by and registered with the Canadian Health Information Management Association and have completed at minimum a 2-year diploma program through an accredited postsecondary institution. ${ }^{21}$

We invited coders who were registered with the (former) Health Information Management Association of Alberta to participate in the study. We also searched LinkedIn and asked key informants - including leadership at the provincial health authority and site-specific health information management supervisors - and interview participants to forward study invitation materials on to their coder colleagues.

Together, 2 study investigators (K.L.T., K.L.) conducted a single 60-minute semistructured interview with each participant, either in person or over the telephone as per the participant's preference. K.L.T. is a physician and research fellow, and K.L. is a PhD candidate and research associate with experience in qualitative methodology. The interviews were conducted between September 2015 and March 2016 at the University of Calgary. The semistructured interview guide covered 5 main domains: 1) education and training, 2) written chart documentation, 3) coding standards and ICD-10, 4) workload and 5) work environment. All interviews were audio-recorded, anonymized and professionally transcribed. Both investigators recorded field notes during the interviews. Transcripts were not returned to study participants, and no feedback was provided on study findings.

\section{Data analysis}

We conducted data analysis using thematic content analysis. ${ }^{22}$ As the focus of this study was neither to form nor to explore complex theory but, rather, to identify and describe barriers experienced by coders, we adopted a qualitative descriptive approach. ${ }^{23,24}$ Two study investigators (K.L.T., K.L.) independently performed line-by-line open coding of each transcript and identified codes relevant to the research objective. Transcripts were reanalyzed as new codes emerged, through constant comparison and iterative review. The investigators interpreted codes through regular meetings, at which coding differences were discussed. Codes were organized into wider themes that captured overarching barriers reported by coders. Themes were derived from the data rather than identified in advance. Data saturation was reached after the analysis of about 20 interview transcripts, when no new themes were further identified. However, as analysis occurred alongside interviews, scheduled participants were still interviewed to refine our themes and to explore new perspectives in different coding settings.

\section{Ethics approval}

Ethics approval was obtained from the University of Calgary Conjoint Health Research Ethics Board.

\section{Results}

Twenty-eight coders were recruited and interviewed, with no losses to follow-up. The median age was 46 years. Most (25 [89\%]) were female, and two-thirds (19 [68\%]) worked in an urban setting with a minimum population of 100000 . The mean number of years of coding experience was 14.8 (standard deviation 9.7) (Table 1).

Five main themes emerged regarding the role that physicians play in contributing to barriers to coding of high-quality administrative data: 1) coders are limited in their ability to add to, modify or interpret physician documentation, which supersedes all other chart documentation, 2) incomplete and nonspecific documentation by physicians, 3) errors, inaccuracies and discrepancies in documentation, 4) physicians and coders use different terminology and 5) there is a communication divide between coders and physicians.

Table 1: Baseline coder demographic and job characteristics

\begin{tabular}{|c|c|}
\hline Characteristic & $\begin{array}{c}\text { No. }(\%) \text { of } \\
\text { participants } \\
n=28\end{array}$ \\
\hline \multicolumn{2}{|l|}{ Age, yr } \\
\hline Mean \pm SD & $46.6 \pm 11.8$ \\
\hline Median (IQR) & $46(38-54)$ \\
\hline Range & $22-71$ \\
\hline Female & $25(89)$ \\
\hline Working in urban setting & $19(68)$ \\
\hline Working full-time & $18(64)$ \\
\hline Supervisory position & $3(11)$ \\
\hline \multicolumn{2}{|l|}{ Primary coding responsibilities $(n=26) \dagger$} \\
\hline Inpatient data & $10(36)$ \\
\hline $\begin{array}{l}\text { Outpatient data including emergency } \\
\text { department visits, day surgical } \\
\text { procedures, clinic visits }\end{array}$ & $5(18)$ \\
\hline Both inpatient and outpatient & $11(39)$ \\
\hline $\begin{array}{l}\text { Required to perform non-coding-related } \\
\text { activities }\end{array}$ & $7(25)$ \\
\hline Length of experience, mean $\pm S D$, yr & $14.8 \pm 9.7$ \\
\hline
\end{tabular}




\section{Coders are limited in their ability to add to, modify or interpret physician documentation, which supersedes all other chart documentation (Box 1)}

Because physicians are the primary health care providers who diagnose and treat the patient's medical condition(s), their documentation takes priority over all other documentation in the process of coding, even when discrepancies exist between their documentation and other supporting documentation (e.g., laboratory or radiologic reports). These supporting documents may be used only to increase the specificity of diagnostic codes and cannot be interpreted by coders outside of this context. ${ }^{25}$ For example, coders cannot code "urinary tract infection" if the physician does not document this diagnosis, even if microbiology reports indicate the presence of pathogenic bacteria on urine culture and antibiotics are prescribed. All participants agreed that the physician is the ultimate source of "truth" in the clinical chart, recognizing that they themselves have limited training in medicine and physiology compared to physicians and that their coding standards have strict guidelines that prioritize documentation by physicians. ${ }^{26}$

\section{Incomplete and nonspecific documentation by physicians (Box 2)}

One participant estimated that discharge summaries were missing in $80 \%$ of charts, which severely limits the coder's ability to code medical information. Furthermore, nearly all participants noted lack of specificity in physician documentation as a major barrier to coding chart information. Common issues described were that physicians do not routinely document the "Most Responsible Diagnosis" (a mandatory field for coders, defined as the diagnosis that contributed most to the patient's length of hospital stay), ${ }^{25}$ do not document the cause of disease even when it is known, do not link related diagnoses (e.g., chronic kidney disease and hypertension) and do not provide sufficient information regarding the timing of diagnoses (e.g., preexisting versus complication arising following the hospital stay). Participants indicated that, although ICD-10 can capture substantial diagnostic specificity, the codes ultimately used were often nonspecific owing to nonspecific physician documentation. Participants attributed nonspecificity in documentation partially to the physician's lack of awareness of the coding process and standards: "If [the physicians] knew that I needed certain specifics, they might be more detailed in their recording in the documentation. If [they] don't know, [they're] not going to ... record it" (interview 21).

\section{Errors, inaccuracies and discrepancies in documentation (Box 3)}

Participants described frequent discrepancies in documentation, which may be the result of uncorrected errors in physician documentation. For example, 1 participant described the interchangeable use of lower versus upper respiratory tract infections in progress notes, although these entities are distinct. Another source of discrepancy may be clinical uncertainty, given that the formulation of diagnoses requires clinical judgment, which is inherently subjective. Discrepancies
Box 1: Supporting quotations for theme 1: Coders are limited in their ability to add to, modify or interpret physician documentation, which supersedes all other chart documentation

"We read all the physicians' notes. We don't really go with the nursing notes. We're supposed to go by what the physician says. He may say congestive heart failure throughout the chart and then he may decide on something else for the most responsible diagnosis." (interview 1)

"If the doctor stated in the discharge summary that the patient had this kind of infection even though you look in the lab [reports] and they don't make a mention [of it] in there, we go by the doctor. We can't question it because we're not doctors so we have to go by what they document. ... The doctors - they're the beginning and the end of it." (interview 6)

"Our guidelines say if [the Most Responsible Diagnosis is] not clearly documented, we're not allowed to assume. We're not doctors. We have 2 years of schooling and 5 months of training." (interview 10)

"[We] have to go with what the physician documented [even if we disagree with it]. We're not doctors. We're not there. We haven't laid eyes on these people. We're only seeing what's on paper." (interview 12)

"We also [have] our own standards. One [example] is $\downarrow$ hemoglobin or $\downarrow \mathrm{Na}$. We know what they're trying to say, but I can't capture it because [they] didn't say anemia or hyponatremia." (interview 20)

"We can't use lab reports, pathology reports, diagnostic imaging reports just to formulate our own opinion. We have to have the physician-stated diagnosis, and then we can use those other reports for additional information." (interview 24)

Box 2: Supporting quotations for theme 2: Incomplete and nonspecific documentation by physicians

"[Physicians] never really say what's the most responsible [diagnosis]. I just hope that I pick the right one. If it looks like they treated the congestive heart failure and that seemed to be the most significant of the 5 things [the patient] came in with, then l'll pick that one, but sometimes I think we're actually doing, you know, a job that's really not ours, but you need to make a choice so I do." (interview 5)

"I mean, even though the [coding] rubric might have 7 or 8 levels of specificity for a particular type of problem, you're probably going to end up going with 'unspecified' because a physician won't document it to that level. For example - pancreatitis: is it idiopathic, is it alcohol-induced, is it sclerosis? Is it chronic recurrent? [ls it] acute? They're just calling it pancreatitis all the way through. ... So in the end, we'll just go with pancreatitis unspecified." (interview 12) "I would say more often than not there is no discharge summary." (interview 13)

"The other thing that I would like [from physicians] is detail. Clarify whether a diagnosis is acute or chronic. Clarify whether that diabetes is type 1 or type 2.... It used to be insulindependent and non-insulin-dependent long before it used to be juvenile and adult. That's gone out the window. [I would like] for doctors to stay current [with the terminology] and to be as specific as possible. Say that pancreatitis was alcohol-related. Let's not compare an acute [case of] pancreatitis that happened without any provocation or preexisting condition to one that had a reason [for it]." (interview 14)

"When you're working on these charts and you see that some of these diagnoses could have been a lot [more specific, I think that is where] the doctors should be made more aware of that. I think that's at the teaching level." (interview 17) 
may also be due to documentation of the patient's progress in hospital by different levels of medical trainees. Participants described uncertainty as to which notes to trust, especially when attending physicians do not supplement or clarify trainee notes, merely "[coming to] scribble 'Agree' and a signature" (interview 23). In the face of errors and discrepancies, participants indicated that they code the diagnosis specified by the physician as required by their coding standards: "We're supposed to go with what the doctors say, so we do, but it just looks kind of funny" (interview 1).

\section{Physicians and coders use different terminology (Box 4)}

Although significant efforts have been made to ensure that the ICD-10 classification system reflects clinical terminology, discrepancies remain. That is, physicians make diagnoses that often do not correlate exactly to the diseases captured by ICD-10, as there is no standard terminology that physicians use or from which they "pick" a diagnosis. In contrast, coders are taught dis-

\section{Box 3: Supporting quotations for theme 3: Errors,} inaccuracies and discrepancies in documentation

"There's times when [physicians will] interchange [upper respiratory tract infection] and [lower respiratory tract infection]. They're completely separate, different systems, but throughout the chart, it'll be interchanged. We don't know which one is the real one. They said them both, we'll code them both." (interview 3)

"Our ... doctors ... will copy and paste [from previous discharge summaries]. So then the diagnosis that I'm actually reading is from the previous hospital [visit], and I really shouldn't be using that as my Most Responsible Diagnosis." (interview 4)

"[The doctors] just say, basically, no [postoperative] complications. When you actually go and pick and look through all the progress notes, there's tons of complications. [The discharge summaries are] very poorly done, a lot of them." (interview 9)

"One doctor will say like, 'Okay. This guy has a [transient ischemic attack]' and the other [says], 'No, no, he has a stroke,' and then you get to the [computerized tomography scan] and it says 'No, he's fine.' Which one do I go by?" (interview 21)

"It's really difficult to weed out what is good information and what's not. ... You've got clinical clerks, which from what I understand is medical students; they're not residents yet, they're clerks. You've got them writing notes, you've got residents writing notes. You're not sure what information you can use. You're not sure what you can trust because they'll write 6 pages. They'll write 4 different differential diagnoses, and the doctor will come and scribble 'Agree' and a signature, and you have to somehow come up with your codes from there." (interview 23)

"So throughout the progress notes, maybe l'll have sepsis, sepsis, sepsis. I mean, 15 days' worth of sepsis and then l'll get the discharge summary back 4 months later and it's dehydration. No mention of sepsis. Then we're sitting here, like, 'Well, did the patient have sepsis? Was that student doctor dreaming, or [was he] just thinking the patient was septic?' I mean, you literally don't know what to do." (interview 24)

"The documentation [can be] conflicting, especially in obstetrics. The nurses could write one thing and the doctors could write another in the discharge summary. That's the hardest part, is when it doesn't match." (Interviewer: "So what do you do in that case?") "Well, the doctor trumps, right?" (interview 25) eases only based on ICD-10 and can choose diagnoses only from this list. For example, a participant described a situation in which a physician diagnosed a specific type of interstitial lung disease but did not use the same terminology as ICD-10. As a result, the coder was unable to identify the disease as a type of interstitial lung disease but, rather, classified it under "pneumonia." Therefore, even when a clinical diagnosis could theoretically be accurately captured by ICD-10 with great specificity, coders are not able to assign the correct codes or are forced to use nonspecific or symptom-based diagnostic codes, unless the exact ICD-10 terminology is used by physicians.

\section{There is a communication divide between coders and physicians (Box 5)}

The coding standards clearly state that when there is uncertainty about coding owing to lack of documentation, coders should clarify these questions with the responsible physician. ${ }^{25}$ Although participants working in rural settings described a good working relationship with physicians, none of those work-

\section{Box 4: Supporting quotations for theme 4: Physicians and} coders use different terminology

"I had a chart today that I was working on and the patient came in cardiogenic shock and he was on [extracorporeal membrane oxygenation]. The discharge summary said that it was due to he had nonischemic cardiomyopathy due to alcohol toxicity. Well, that didn't seem right to me so ... we are going to look in the progress notes. The better, more specific code is alcohol cardiomyopathy because, for us, if we see toxicity, we're coding in overdose, which doesn't fit, right?" (interview 9)

"I had a chart yesterday. Patient came in for an elective [surgical procedure] and they ran some blood work day of admission and her clotting was all whacky. There's no way they're going to do any sort of incision because she'll probably bleed out in the [operating room]. They had hematology involved trying to figure out why her platelets were so whacky, and she ended up spending 5 days in the hospital. Her procedure was cancelled and she was sent home. What was wrong with her? Nobody really said. How do you code that? How do you tell that story? ... I couldn't really assign a diagnosis code to the fact that her platelets were whacky because it wasn't described as thrombocytopenia. It wasn't described as some sort of blood dyscrasia. It was just this lab result was suspect." (interview 12)

"I went to the [Canadian Institute for Health Information] and I put the words, the terms in there, and nothing came up in there. Then I put as many terms as I possibly could into [International

Classification of Diseases] and it's not there. So I just have to pull some really generic one that just says disorder of the lung, not yet diagnosed. Yeah. I think it was what they call ground glass in the lungs. There was ... no code for it." (interview 15)

"When is the last time you ever saw [a physician] even look at an [International Classification of Diseases] manual? Never. Do they even know that coding standards exist? I don't think so. I don't think it's a big push when they're learning to be physicians." (interview 16)

"In our abstracting system, if you type in urosepsis or if you type in [urinary tract infection], it goes to the same code, but in recent conversations with our physicians, we have been hearing that urosepsis means the patient has sepsis with the urinary tract infection." (interview 20) 
ing in urban settings felt they could routinely approach physicians. Rural coders attributed the ability to communicate with physicians to the smaller size of the hospital, where they "get to know everybody there" (interview 11). In contrast, urban coders indicated that the doctors are "surprised that we even exist" (interview 13). Several participants working in urban settings gave examples of physicians' seeking them out in the health records department to "teach them" about coding (interview 21). This occurred infrequently and generally only when a physician perceived his or her resources as threatened or when the physician realized that coded data were nonspecific and did not reflect actual patient care. These types of interaction resulted in coders' feeling blamed for quality issues around coded data.

\section{Box 5: Supporting quotations for theme 5: There is a} communication divide between coders and physicians

"I think there should be more emphasis put on ... holding up [the physician's] end of giving us the documentation. I know it's really hard to do, and it doesn't seem to ever happen. ... Whatever we do as coders is a lot of the time a guessing game because [the physicians are] just not giving [the information] to us. And they don't really care, too. As far as they're concerned, it's just stats, and they don't really care." (interview 8 )

"The physicians? They're awesome. Yeah. They'll help; they're so quick and easy. I have sent [charts] back to the physician or went to see the physician, like, "Hey. This doesn't make sense to me from a coding perspective," and [the physician will] give me the answer, you know, which way to go with it. I mean, that's the benefit of being a smaller facility, too. You do get to know everybody there, right?" (interview 11)

"[The physician] contacted the coding team ... so we had this meeting. She pulled up this whole thing about [International Classification of Diseases, 9th Revision] and all this information in the [United] States. We're trying to explain to her, 'Okay, we're ICD-10.' She had the perception that coding was only matching her diagnosis with a code. She had a good understanding of coding in a general sense, but she didn't know that there's a lot of really hard mandated rules. She had no idea about clusters. She had no idea about typings. But then she continued on and said 'You guys are not qualified to code.' Like she was right in the sense that she's the only single person in that room that could make ... a diagnosis ... and care for a patient, but she was the single person in that whole room that was not qualified to code.

You know, we've all gone to school ... we've passed the national exam. ... Even now ... they're thinking of actually extending [the training for] coding ... just because they feel that it takes so much resources to train [people] once they get hired. ... [It's] bothered me ever since that [the physician] left with that misconception about us. You know, we don't just come straight off the street." (interview 21)

"I really think that coders need to have more ... ability to confer with the physicians. Ever since I started coding, I remember it's always been impressed upon me that God forbid should you ever, ever disturb [physicians] by asking them for something, you know. The standards, though, say in a couple of areas [to] return the chart to the physician. We all laugh because ... well, we can't. Obviously, physicians are busy. We can't bother them, but at the same time, if they don't understand our issues that we're running into with their documentation, how is anything ever going to change?" (interview 24)

"The doctors don't even know why they need to have their charts done on time. They don't see that that can affect funding for a facility or a clinic that they're going to implement. They don't see the importance of it." (interview 28)
Participants described a desire to be able to openly communicate with physicians. Several participants described the presence of a "physician champion" at a single urban hospital, a liaison to bring coder concerns to the attention of physicians. Participants described physician champions as "our foot in the door" (interview 4) in improving the quality of coding and expressed a desire for such a role at every health care facility.

\section{Interpretation}

Despite its importance, physician documentation of patient care remains incomplete, nonspecific and replete with errors and discrepancies. We also discovered issues beyond documentation that act as barriers to producing high-quality data, including the disconnect between clinical diagnoses made by physicians and standard disease classifications in ICD-10, and the lack of interaction between physicians and coders to reconcile these problems.

The literature describes coder- and organizational-level barriers that affect the quality of administrative data, such as deficiencies in training and insufficient human resources. ${ }^{11-17}$ However, coders constitute only part of the equation in coding quality; their output depends on the quality of the input, which is physician documentation of a patient's course in hospital. As physician researchers, we acknowledge that many of the identified physician-related barriers are structural and systemic, extending beyond the control of physicians alone. For example, the problem of imprecise documentation stems from the fact that chart information, when translated to administrative data, is being used outside the scope of its primary purpose. Charts are used by health care providers to document the patient's clinical course and also as a communication tool. ${ }^{27,28}$ Physicians also write notes as a method of "sensemaking," or a way to systematically synthesize information required for decision-making in providing patient care..$^{29,30}$ Therefore, documentation may lack specificity not because physicians are making imprecise diagnoses but, rather, to optimize efficiency; their notes are meant to interpret the information in the chart, not to duplicate it. Lack of specificity is therefore a complex issue that cannot be addressed solely by asking physicians to increase the level of detail provided in their notes. ${ }^{31}$ There may be a role for interventions at the health information level, such as broadening the power of coders to make certain decisions or corrections in light of clear documentation errors and deemphasizing physician documentation as the only or ultimate source of truth.

A second systemic issue concerns differences in terminology: clinical diagnoses made by physicians do not always correlate, at a 1:1 ratio, to diseases in ICD-10. Whereas diseases, or pathologic conditions that affect the normal functioning of the body, are abstract (e.g., arteriosclerosis), diagnoses are more concrete, as they may be symptom-based (e.g., angina) or a series of facts that are not in themselves diseases (e.g., post-angiography). ${ }^{32}$ This is a fundamental problem of translating one "language" that is relevant for clinical care to another "language" of standardized disease classifications that is important for epidemiology and health care planning. Neither physicians nor coders can solve this issue at an individual 


\section{OPEN}

level, as paradigmatic changes to international disease classification systems are required.

\section{Limitations}

There are several limitations to our study. First, we interviewed coders only, with no input from physicians. Although coders were well equipped to describe barriers to coding and the physician's contributions to these barriers, a future study that specifically explores physician views on why these barriers exist is needed to develop interventions to mitigate them. Second, some countries do not have accredited coding specialists to code chart information into coded data. This may limit the transferability of our findings, although we believe that our conclusions hold given the universal role of physicians in health care provision and that our focus is on physician-related (rather than coder-related) barriers to high-quality administrative data.

\section{Conclusion}

Despite the limitations of our study and the acknowledged fundamental issues inherent to coded data, there are changes that physicians can make to improve the quality of coded data. First, they have a responsibility to complete accurate and comprehensive discharge summaries in a timely manner; this is important not only for coding but also to serve as a useful communication tool across health care providers. In progress notes, we recommend that physicians outline the timing and acuity of medical diagnoses, such as whether a condition is preexisting versus arising since hospital admission, as quality indicators depend on this information. Although there is a fine balance between detailed documentation and efficiency, highly specific notes are helpful not just for coders but also for continuity of care and communication purposes. Furthermore, charts also serve as legal documents, and a better appreciation of the gravity of this may help to minimize errors in documentation. Notes from trainees should be regularly checked for accuracy, with errors corrected. Last, a shift in physician culture to respect, value and acknowledge the contributions of coders as an essential part of the health care team is an important first step in mitigating many of the barriers to producing high-quality of administrative data.

\section{References}

1. O'Malley KJ, Cook KF, Price MD, et al. Measuring diagnoses: ICD code accuracy. Health Serv Res 2005;40:1620-39.

2. Roos LL Jr, Nicol JP, Cageorge SM. Using administrative data for longitudinal research: comparisons with primary data collection. 7 Chronic Dis 1987;40:41-9.

3. Roos LL, Gupta S, Soodeen RA, et al. Data quality in an information-rich environment: Canada as an example. Can 7 Aging 2005;24(Suppl 1):153-70.

4. Burns EM, Rigby E, Mamidanna R, et al. Systematic review of discharge coding accuracy. 7 Public Health (Oxf) 2012;34:138-48.

5. Gologorsky Y, Knightly JJ, Lu Y, et al. Improving discharge data fidelity for use in large administrative databases. Neurosurg Focus 2014;36:E2.

6. Quan H, Parsons GA, Ghali WA. Validity of information on comorbidity derived from ICD-9-CCM administrative data. Med Care 2002;40:675-85.

7. Quan H, Parsons GA, Ghali WA. Validity of procedure codes in International Classification of Diseases, 9th revision, clinical modification administrative data. Med Care 2004;42:801-9.

8. CIHI data quality study of Ontario emergency department visits for fiscal year 2004-2005 - executive summary. Ottawa: Canadian Institute for Health Information; 2008.

9. Martin BJ, Chen G, Graham M, et al. Coding of obesity in administrative hospital discharge abstract data: accuracy and impact for future research studies. BMC Health Serv Res 2014;14:70.
10. CIHI data quality study of the 2009-2010 Discharge Abstract Database. Ottawa: Canadian Institute for Health Information; 2012:115.

11. Bramley $M$, Reid B. Evaluation standards for clinical coder training. HIM 7 2007;36:20-30.

12. Hennessy DA, Quan H, Faris PD, et al. Do coder characteristics influence validity of ICD-10 hospital discharge data? BMC Health Serv Res 2010;10:99.

13. Santos S, Murphy G, Baxter K, et al. Organisational factors affecting the quality of hospital clinical coding. HIM 7 2008;37:25-37.

14. Walker RL, Hennessy DA, Johansen H, et al. Implementation of ICD-10 in Canada: How has it impacted coded hospital discharge data? BMC Health Serv Res 2012;12:149.

15. Callen J, Hines N, Rust J, et al. Is coding in theatres a viable option? A review of current theatre coding process at Royal Prince Alfred Hospital. ACORN 7 1998;11:34-8.

16. Lorenzoni L, Da Cas R, Aparo UL. The quality of abstracting medical information from the medical record: the impact of training programmes. Int $f$ Qual Health Care 1999;11:209-13.

17. Lorenzoni L, Da Cas R, Aparo UL. Continuous training as a key to increase the accuracy of administrative data. 7 Eval Clin Pract 2000;6:371-7.

18. McKenzie K, Walker S. The Australian coder workforce 2002: a report of the National Clinical Coder Survey. Sydney (AU): University of Sydney; 2003.

19. Goodman LA. Snowball sampling. Ann Math Stat 1961;32:148-70.

20. Heckathorn DD. Snowball versus respondent-driven sampling. Sociol Methodol 2011;41:355-66.

21. Accredited HIM schools. London (ON): Canadian Health Information Management Association; 2017. Available: https://www.echima.ca/cchim/him -program (accessed 2017 May 29).

22. Braun V, Clarke V. Using thematic analysis in psychology. Qual Res Psychol 2006;3:77-101.

23. Vaismoradi M, Turunen H, Bondas T. Content analysis and thematic analysis: implications for conducting a qualitative descriptive study. Nurs Health Sci 2013;15:398-405.

24. Colorafi KJ, Evans B. Qualitative descriptive methods in health science research. HERD 2016;9:16-25.

25. Canadian coding standards for version 2015 ICD-10-CA and CCI. Ottawa: Canadian Institute for Health Information; 2015.

26. Canadian coding standards for version 2009 ICD-10-CA and CCI. Ottawa: Canadian Institute for Health Information; 2009.

27. Pearce PF, Ferguson LA, George GS, et al. The essential SOAP note in an EHR age. Nurse Pract 2016;41:29-36.

28. Lenert LA, Sakaguchi FH, Weir CR. Rethinking the discharge summary: a focus on handoff communication. Acad Med 2014;89:393-8.

29. Mamykina L, Vawdrey DK, Stetson PD, et al. Clinical documentation: composition or synthesis? 7 Am Med Inform Assoc 2012;19:1025-31.

30. Weed LL. Medical records that guide and teach. $N$ Engl 7 Med 1968;278: 593-600.

31. Russo R. Documentation and data improvement fundamentals. In: Proceedings from the 2004 IFHRO Congress \& AHIMA Convention; 2004 Oct.; Washington. Chicago: American Health Information Management Association; 2004. Available: http://bok.ahima.org/doc?oid=60174\#.WVPNamdX7IU (accessed 2016 June 23).

32. Surjan G. Questions on validity of International Classification of Diseasescoded diagnoses. Int 7 Med Inform 1999;54:77-95.

Affiliations: Departments of Medicine (Tang) and Community Health Sciences (Lucyk, Quan), University of Calgary, Calgary, Alta.

Contributors: Karen Tang and Kelsey Lucyk collected and analyzed the data and reviewed the codes generated to develop themes and subthemes to address the research questions. Karen Tang drafted the manuscript. All of the authors conceived of the study and were involved in the development of the study design, interpreted the data and themes, critically revised the manuscript for important intellectual content, approved the final version to be published and agreed to act as guarantors of the work.

Funding: This study was funded by a Catalyst Grant from the O'Brien Institute for Public Health, University of Calgary. The funder was not involved in the study conception or design, or data collection, analysis or interpretation. Karen Tang is supported by fellowship awards from the Canadian Institutes of Health Research and Alberta Innovates - Health Solutions. Kelsey Lucyk is supported by an Alberta Innovates - Health Solutions Graduate Studentship and an Achievers in Medical Sciences recruitment scholarship, University of Calgary. Hude Quan receives salary support from Alberta Innovates - Health Solutions.

Supplemental information: For reviewer comments and the original submission of this manuscript, please see www.cmajopen.ca/content/5/3/ E617/suppl/DC1. 